\title{
The Existence of Near Generalized Balanced Tournament Designs
}

\author{
Chengmin Wang, Jie Yan* \\ School of Science, Jiangnan University, \\ Wuxi 214122, China \\ Submitted: May 15, 2011; Accepted: Apr 29, 2012; Published: Jun 6, 2012 \\ Mathematics Subject Classifications: 05B05, 94B25
}

\begin{abstract}
In this paper, we complete the existence of near generalized balanced tournament designs (NGBTDs) with block size 3 and 5. As an application, we obtain new classes of optimal constant composition codes.
\end{abstract}

Keywords: near generalized balanced tournament design; constant composition codes; optimal; existence

\section{Introduction}

Let $X$ be a set of $v$ points, and $\mathcal{A}$ a collection of subsets (called blocks) of $X$. A $(v, k, \lambda)$ balanced incomplete block design (BIBD), or a $(v, k, \lambda)$-BIBD, is a pair $(X, \mathcal{A})$ such that any pair of distinct points of $X$ occurs in precisely $\lambda$ blocks. $\mathrm{A}(k m+1, k, k-1)$ $\operatorname{BIBD}(X, \mathcal{A})$ is called a near generalized balanced tournament design (NGBTD), or an $\operatorname{NGBTD}(k, m)$ in short, if its blocks can be arranged into an $m \times(k m+1)$ array in such a way that

(1) the blocks in each column form a partial parallel class which partition $X \backslash\{x\}$ for some point $x \in X$;

(2) each point of $X$ is contained in precisely $k$ cells of each row.

By the definition, any NGBTD can be identified with its corresponding block array defined above.

NGBTDs are a generalization of odd balanced tournament designs (OBTDs). Particularly, an $\operatorname{NGBTD}(2, m)$ is an $\operatorname{OBTD}(m)$ whose existence was completed in [5]. Lamken [4] almost finished the existence of $\operatorname{NGBTD}(3, m)$ with four possible exceptions. Recently, Shan [8] presented a nearly complete solution for the existence of $\operatorname{NGBTD}(k, m)$ 's for

\footnotetext{
${ }^{*}$ Corresponding author. Email: jyan7906@yahoo.com.cn
} 
$k=4$ and 5 with four possible exceptions. We collect the existence of $\operatorname{NGBTD}(k, m)$ 's for $2 \leqslant k \leqslant 5$ as follows.

Theorem 1. ([4, 5, 8])

(1) There exists an $\operatorname{NGBTD}(2, m)$ for any positive integer $m$;

(2) There exists an $\operatorname{NGBTD}(3, m)$ for any positive integer $m$ and $m \notin\{3,38,39,118\}$;

(3) There exists an $\operatorname{NGBTD}(4, m)$ for any positive integer $m$;

(4) There exists an $\operatorname{NGBTD}(5, m)$ for any positive integer $m$ and $m \notin\{15,32,40,45\}$.

A group divisible design of block size $k$ and index $\lambda$, or a $(k, \lambda)$-GDD, is a triple $(X, \mathcal{G}, \mathcal{B})$ where $X$ is a finite set of (points), $\mathcal{G}$ is a partition of $X$ into subsets (called groups), and $\mathcal{B}$ is a set of subsets of size $k$ (called blocks) of $X$, such that every pair of points from distinct groups occurs in exactly $\lambda$ blocks, and any pair of points from the same group occur in no block. The type of the GDD is defined to be the multiset $T=\{|G|: G \in \mathcal{G}\}$, which is usually denoted by an "exponential" notation: a type $g_{1}^{u_{1}} g_{2}^{u_{2}} \cdots g_{s}^{u_{s}}$ means $u_{i}$ occurrences of $g_{i}$ for $1 \leqslant i \leqslant s$.

A set of blocks of a $\operatorname{GDD}(X, \mathcal{G}, \mathcal{B})$ is called a partial $\alpha$-parallel class over $X \backslash S$ if each point of $X \backslash S$ occurs in exactly $\alpha$ blocks, while any point of $S$ occurs in no block. If $S=\emptyset$, it is called an $\alpha$-parallel class over $X$. Whenever $\alpha=1$, we simply say a (partial) parallel class, instead of a (partial) $\alpha$-parallel class. A GDD is called resolvable if its blocks can be partitioned into parallel classes.

We need a special type of GDDs, called frame generalized doubly resolvable packings (FGDRPs), which was first introduced in [11] to construct optimal constant composition codes.

Let $(X, \mathcal{G}, \mathcal{B})$ be a $(k, k-1)$-GDD of type $g^{u}$ where $g=k h$. Suppose that the group set $\mathcal{G}=\left\{G_{1}, G_{2}, \cdots, G_{u}\right\}$. Define

$$
\begin{aligned}
& R_{i}=\{(i-1) h+j: j=1, \cdots, h\} \\
& C_{i}=\{(i-1) k h+j: j=1, \cdots, k h\}
\end{aligned}
$$

for $1 \leqslant i \leqslant u$. The blocks of the GDD can be arranged into an $h u \times g u$ array $F$ which satisfies the following properties:

(1) Each cell of $F$ is either empty or contains a block of $\mathcal{B}$;

(2) Let $F_{t}$ be the subarray indexed by the elements of $R_{t}$ and $C_{t}$. Then $F_{t}$ is empty for $t=1,2, \cdots, u$, i.e., the main diagonal of $F$ consists of $u$ empty subarrays of size $h \times k h$;

(3) For any $x \in R_{i}(1 \leqslant i \leqslant u)$, the blocks in row $x$ form a partial $k$-parallel class over $X \backslash G_{i}$;

(4) For any $y \in C_{j}(1 \leqslant j \leqslant u)$, the blocks in column $y$ form a partial parallel class over $X \backslash G_{j}$.

Then we refer to this GDD as an $\operatorname{FGDRP}\left(k, g^{u}\right)$. Actually, FGDRPs can be defined in a more general way in which all the groups are not necessarily the same size. Nevertheless, we use the definition here for our purpose. The interest reader may refer to $[10,11]$ for more details on FGDRPs.

In this note, we completely establish the existence of $\operatorname{NGBTD}(k, m)$ 's for $k=3$ and 5 by removing all the remaining cases in Theorem 1. We also present an application of NGBTDs to optimal constant composition codes. 


\section{The Existence of NGBTDs with Block Size 3}

In this section, we give a complete solution to the existence of NGBTDs with block size 3 .

Theorem 2. [8, 9] Suppose that there exists an $F G D R P\left(k, g^{u}\right)$ where $g=k h$. Let $w$ be any nonnegative integer. If there exists an $\operatorname{NGBTD}(k, h+w)$ which contains an $N G B T D(k, w)$ as a subarray, then an $N G B T D(k, h u+w)$ exists.

Theorem 3. $[9,10]$ Let $g$ and $u$ be positive integers with $g \equiv 0$ (mod 3) and $u \geqslant 5$. Then an $\operatorname{FGDRP}\left(3, g^{u}\right)$ exists except possibly for $(g, u) \in$ $\{(6,15),(9,18),(9,28),(9,34),(30,15)\}$.

Theorem 4. For any integer $m \geqslant 1$ and $m \neq 3$, there exists an $\operatorname{NGBTD}(3, m)$. There does not exist an $N G B T D(3,3)$.

Proof : By Theorem 3, there exists an $\operatorname{FGDRP}\left(3,3^{m}\right)$ for any $m \geqslant 5$. An $\operatorname{NGBTD}(3,1)$ exists trivially with all the subsets containing any three points as blocks. Then we apply Theorem 2 with $k=3, h=1, u=m, w=0$ to obtain an $\operatorname{NGBTD}(3, m)$ for any $m \geqslant 5$.

We know that there does not exist an $\operatorname{NGBTD}(3,3)$ definitely by an exhaustive search with the aid of a computer.

Combining with Theorem 1, we complete the proof.

Remark: Here we have fixed the four possible exceptions in [4] and complete the existence of $\operatorname{NGBTD}(3, m)$ 's. Moreover, Theorem 4 actually provides an alternative proof for the existence of $\operatorname{NGBTD}(3, m)$ 's.

\section{The Existence of NGBTDs with Block Size 5}

This section serves to complete the existence of NGBTDs with block size 5 , by removing the four outstanding cases in Theorem 1.

Lemma 5. There exists an $\operatorname{NGBTD}(5,15)$.

Proof : We construct the desired NGBTD on the Abelian group $X=\mathbb{Z}_{19} \times \mathbb{Z}_{2} \times \mathbb{Z}_{2}$. Here we write the element $(a, b, c)$ of $X$ as $\underline{a} b c$ for brevity. The following blocks form a partial parallel class which partitions $X \backslash\{\underline{18} 11\}$.

$$
\begin{aligned}
& \{\underline{0} 01, \underline{0} 00, \underline{0} 10, \underline{0} 11, \underline{100}\} \quad\{\underline{16} 01, \underline{3} 01, \underline{12} 10, \underline{9} 10, \underline{14} 01\} \quad\{\underline{14} 11, \underline{13} 11, \underline{701}, \underline{17} 01, \underline{2} 00\}
\end{aligned}
$$

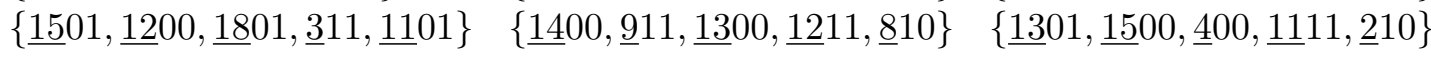

$$
\begin{aligned}
& \{\underline{101}, \underline{3} 00, \underline{10} 11, \underline{1100}, \underline{17} 11\} \quad\{\underline{1} 11, \underline{4} 01, \underline{6} 11, \underline{9} 01, \underline{14} 10\} \quad\{\underline{3} 10, \underline{16} 11, \underline{711}, \underline{10} 00, \underline{5} 01\} \\
& \{\underline{5} 00, \underline{6} 10, \underline{12} 01, \underline{2} 11, \underline{1700}\} \quad\{\underline{4} 11, \underline{7} 00, \underline{1} 10, \underline{16} 10, \underline{8} 11\} \quad\{\underline{5} 10, \underline{10} 10, \underline{6} 00, \underline{7} 10, \underline{11} 10\}
\end{aligned}
$$

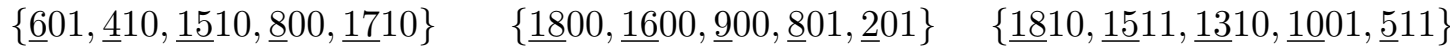

It is easy to check that the desired design is produced by the action of the group $X$ on above partial parallel class. 
Before we move to the next construction, we need the well-known starter-adder method, which is widely used to produce some designs with orthogonal properties (see, for example, $[1,2,5,7,8,13])$.

Let $G$ be an additively Abelian group of order $u$. Let $g=k t$. A starter $S$ for an $\operatorname{FGDRP}\left(k, g^{u}\right)$ over $\mathbb{Z}_{g} \times G$ with groups $G_{x}=\mathbb{Z}_{g} \times\{x\} \quad(x \in G)$ consists of $t$ sets of $k$-tuples (base blocks), $S_{1}, S_{2}, \cdots, S_{t}$, which satisfies the following properties:

(1) For any $i(1 \leqslant i \leqslant t), S_{i}$ contains exactly $u-1$ base blocks $B_{i j}, j=1,2, \cdots, u-1$;

(2) $S=\bigcup_{i=1}^{t} S_{i}$ forms a partition of $\mathbb{Z}_{g} \times(G \backslash\{0\})$ and every element of $\mathbb{Z}_{g} \times(G \backslash\{0\})$ occurs exactly $k-1$ times in the difference list of $S$.

A corresponding adder $A(S)$ for the starter $S$ consists $t$ permutations (not necessarily distinct) of $G \backslash\{0\}$

$$
A\left(S_{i}\right)=\left(a_{i 1}, a_{i 2}, \cdots, a_{i(u-1)}\right), 1 \leqslant i \leqslant t
$$

such that for any $i(1 \leqslant i \leqslant t), \bigcup_{j=1}^{u-1}\left(B_{i j}+\left(0, a_{i j}\right)\right)$ contains exactly $k$ elements (not necessarily distinct) from each group $G_{x}$ for $x \in G \backslash\{0\}$ and no element of $G_{0}$.

Theorem 6. [10] If there exists a starter-adder pair $(S, A(S))$ for an $F G D R P\left(k, g^{u}\right)$ over $\mathbb{Z}_{g} \times G$ defined above, then there exists an $F G D R P\left(k, g^{u}\right)$.

Lemma 7. There exists an $\operatorname{FGDRP}\left(5,25^{9}\right)$.

Proof: We take $\mathbb{Z}_{25} \times G F(9)$ as the point set and $\left\{\mathbb{Z}_{25} \times\{x\} \mid x \in G F(9)\right\}$ as the groups. Let $G F(9)^{*}=G F(9) \backslash\{0\}$. Suppose that $\omega$ is a primitive element of $G F(9)$ satisfying $\omega^{2}=\omega+1$ and $C_{0}^{2}$ is the square residue of $G F(9)^{*}$. We display the starter $S$ and the corresponding adder $A(S)$ in Table 1. Then we apply Theorem 6 to produce the desired design.

Table 1: The starters and corresponding adders for an $F G D R P\left(25^{9}\right)$

\begin{tabular}{|ccc|}
\hline & \multicolumn{1}{c|}{$S$} & $A(S)$ \\
\hline$S_{1}:$ & $\left\{\left(0, \omega^{1}\right),\left(8, \omega^{5}\right),\left(8, \omega^{2}\right),\left(13, \omega^{3}\right),\left(14, \omega^{7}\right)\right\} \cdot(1, h)$ & $(0,1) \cdot(1, h)$ \\
& $\left\{\left(9, \omega^{0}\right),\left(10, \omega^{7}\right),\left(21, \omega^{1}\right),\left(23, \omega^{2}\right),\left(15, \omega^{6}\right)\right\} \cdot(1, h)$ & $(0, \omega) \cdot(1, h)$ \\
$S_{2}:$ & $\left\{\left(2, \omega^{6}\right),\left(11, \omega^{5}\right),\left(12, \omega^{2}\right),\left(14, \omega^{0}\right),\left(15, \omega^{1}\right)\right\} \cdot(1, h)$ & $(0,1) \cdot(1, h)$ \\
& $\left\{\left(5, \omega^{4}\right),\left(6, \omega^{6}\right),\left(17, \omega^{3}\right),\left(20, \omega^{7}\right),\left(24, \omega^{1}\right)\right\} \cdot(1, h)$ & $(0, \omega) \cdot(1, h)$ \\
$S_{3}:$ & $\left\{\left(10, \omega^{0}\right),\left(18, \omega^{1}\right),\left(18, \omega^{6}\right),\left(19, \omega^{3}\right),\left(23, \omega^{7}\right)\right\} \cdot(1, h)$ & $(0,1) \cdot(1, h)$ \\
& $\left\{\left(2, \omega^{1}\right),\left(4, \omega^{3}\right),\left(4, \omega^{6}\right),\left(11, \omega^{2}\right),\left(13, \omega^{4}\right)\right\} \cdot(1, h)$ & $(0, \omega) \cdot(1, h)$ \\
$S_{4}:$ & $\left\{\left(3, \omega^{6}\right),\left(7, \omega^{1}\right),\left(19, \omega^{0}\right),\left(22, \omega^{2}\right),\left(22, \omega^{7}\right)\right\} \cdot(1, h)$ & $(0,1) \cdot(1, h)$ \\
& $\left\{\left(1, \omega^{2}\right),\left(6, \omega^{1}\right),\left(9, \omega^{7}\right),\left(16, \omega^{0}\right),\left(24, \omega^{6}\right)\right\} \cdot(1, h)$ & $(0, \omega) \cdot(1, h)$ \\
$S_{5}:$ & $\left\{\left(1, \omega^{1}\right),\left(12, \omega^{5}\right),\left(16, \omega^{3}\right),\left(17, \omega^{6}\right),\left(20, \omega^{0}\right)\right\} \cdot(1, h)$ & $(0,1) \cdot(1, h)$ \\
& $\left\{\left(0, \omega^{0}\right),\left(3, \omega^{1}\right),\left(5, \omega^{3}\right),\left(7, \omega^{2}\right),\left(21, \omega^{4}\right)\right\} \cdot(1, h)$ & $(0, \omega) \cdot(1, h)$
\end{tabular}

The second type of starter-adder construction is called intransitive starter-adder method, which involves infinite points. The reader may refer to $[2,5,7,8,10,13]$ for more details. 
Let $G F(u-1)$ be the Galois field with $u-1$ elements and $X=\mathbb{Z}_{g} \times(G F(u-1) \bigcup\{\infty\})$ where $g=k t$. Let $G=\mathbb{Z}_{g} \times G F(u-1)$. An intransitive starter $S$ for an $\operatorname{FGDRP}\left(k, g^{u}\right)$ over $X$ with groups $\left\{G_{x}=\mathbb{Z}_{g} \times\{x\} \mid x \in G F(u-1) \bigcup\{\infty\}\right\}$ is defined as a triple $(S, R, C)$ satisfying the following properties.

(1) $S$ consists of $t$ sets of $k$-tuples (base blocks), $S_{1}, S_{2}, \cdots, S_{t}$. For any $i(1 \leqslant i \leqslant t)$, $S_{i}$ contains precisely $u-2$ base blocks, $B_{i j}(1 \leqslant i \leqslant u-2)$, in which there exist exactly $k$ base blocks containing one infinite point each from $G_{\infty}$;

(2) $R$ consists of $t$ base blocks over $G$, denoted by $R_{1}, R_{2} \cdots, R_{t}$, in which each contains no infinite points;

(3) $C$ consists of $t$ base blocks over $G$, denoted by $C_{1}, C_{2} \cdots, C_{t}$, in which each contains no infinite points;

(4) $S \bigcup R$ forms a partition of $X \backslash G_{0}$;

(5) the difference list from the base blocks of $S \bigcup R \cup C$ contains every element of $G \backslash G_{0}$ precisely $k-1$ times, and no element in $G_{0}$.

A corresponding adder $A(S)$ for $S$ consists of $t$ permutations on $G F(u-1) \backslash\{0\}$,

$$
A\left(S_{i}\right)=\left(a_{i 1}, a_{i 2}, \cdots, a_{i(u-2)}\right)(1 \leqslant i \leqslant t)
$$

For any $i(1 \leqslant i \leqslant t)$, the multiset $\bigcup_{j=1}^{u-2}\left(B_{i j}+\left(0, a_{i j}\right)\right) \bigcup\left\{C_{i}\right\}$ contains exactly $k$ elements (not necessarily distinct) from each group $G_{x}$ for $x \in G F(u-1) \backslash\{0\}$ and no element of $G_{0}$.

Theorem 8. [10] If there exists an intransitive starter $(S, R, C)$ over $X$ defined above and a corresponding adder $A(S)$, then there exists an $\operatorname{FGDRP}\left(k, g^{u}\right)$.

Lemma 9. There exists an $\operatorname{FGDRP}\left(5,20^{8}\right)$.

Proof : Let $X=\mathbb{Z}_{20} \times\left(\mathbb{Z}_{7} \cup\{\infty\}\right)$ be the point set and $\left\{\mathbb{Z}_{20} \times\{x\} \mid x \in \mathbb{Z}_{7} \cup\{\infty\}\right\}$ the group set. Here we apply Theorem 8 with $k=5, t=4$. In the following table, we present $S_{i}, R_{i}, C_{i}$ and $A\left(S_{i}\right)$ for $i=1$ and 2 .

\begin{tabular}{|cc||cc|}
\hline$A\left(S_{1}\right)$ & $S_{1}$ & $A\left(S_{2}\right)$ & $S_{2}$ \\
\hline$(0,6)$ & $\{(3,3),(19,6),(0,2),(17,5),(2,4)\}$ & $(0,6)$ & $\{(4,5),(17,4),(9,2),(12,3),(15,6)\}$ \\
$(0,5)$ & $\{-,(7,5),(14,3),(13,1),(4,6)\}$ & $(0,5)$ & $\{-,(15,5),(2,6),(11,3),(19,4)\}$ \\
$(0,4)$ & $\{-,(2,5),(0,4),(9,6),(1,2)\}$ & $(0,4)$ & $\{-,(4,4),(6,6),(12,1),(13,2)\}$ \\
$(0,3)$ & $\{-,(8,1),(3,6),(19,2),(10,5)\}$ & $(0,3)$ & $\{-,(18,3),(11,1),(12,2),(7,6)\}$ \\
$(0,2)$ & $\{-,(16,2),(16,4),(1,6),(8,3)\}$ & $(0,2)$ & $\{-,(3,2),(5,4),(9,3),(16,6)\}$ \\
$(0,1)$ & $\{-,(5,1),(5,5),(10,4),(14,2)\}$ & $(0,1)$ & $\{-,(6,3),(6,5),(14,1),(18,2)\}$ \\
\hline \hline$C_{1}:$ & $\{(4,2),(14,4),(14,3),(0,6),(0,5)\}$ & $C_{2}:$ & $\{(6,3),(5,1),(8,6),(8,2),(7,5)\}$ \\
$R_{1}:$ & $\{(7,4),(11,5),(1,3),(17,1),(0,6)\}$ & $R_{2}:$ & $\{(10,6),(18,1),(15,4),(13,3),(8,2)\}$ \\
\hline
\end{tabular}

For $i=3$ and 4 , let $S_{i}=S_{i-2} \cdot(1,-1), R_{i}=R_{i-2} \cdot(1,-1), C_{i}=C_{i-2} \cdot(1,-1)$ and $A\left(S_{i}\right)=A\left(S_{i-2}\right) \cdot(1,-1)$. Then it is easy to check that $\left(\cup_{i=1}^{4} S_{i}, \cup_{i=1}^{4} R_{i}, \cup_{i=1}^{4} C_{i}\right)$ is an intransitive starter and $A(S)=\cup_{i=1}^{4} A\left(S_{i}\right)$ is the corresponding adder for an $\operatorname{FGDRP}\left(5,20^{8}\right)$. Here the twenty points from $\mathbb{Z}_{20} \times\{\infty\}$ can be distributed to the five blocks of size four in each $S_{i}$ for $1 \leqslant i \leqslant 4$ in an arbitrary way. So we use the symbol "-" to denote any point from $\mathbb{Z}_{20} \times\{\infty\}$. 
Lemma 10. There exists an $\operatorname{FGDRP}\left(5,20^{10}\right)$.

Proof : Here we take $\mathbb{Z}_{20} \times(G F(9) \cup\{\infty\})$ as the point set and $\left\{\mathbb{Z}_{20} \times\{x\} \mid x \in\right.$ $G F(9) \cup\{\infty\}\}$ as the group set. Suppose that $\omega$ is a primitive element of $G F(9)$ satisfying $\omega^{2}=\omega+1$. We apply Theorem 8 with $k=5, t=4$. First we display $S_{1}, R_{1}, C_{1}$ and $A\left(S_{1}\right)$ in the following table.

\begin{tabular}{|cc|}
\hline$A\left(S_{1}\right)$ & $S_{1}$ \\
\hline$(0,1)$ & $\left\{\left(16, \omega^{0}\right),\left(7, \omega^{6}\right),\left(14, \omega^{2}\right),\left(10, \omega^{7}\right),\left(1, \omega^{1}\right)\right\}$ \\
$(0, \omega)$ & $\left\{\left(3, \omega^{0}\right),\left(18, \omega^{1}\right),\left(18, \omega^{2}\right),\left(6, \omega^{3}\right),\left(4, \omega^{7}\right)\right\}$ \\
$\left(0, \omega^{2}\right)$ & $\left\{\left(6, \omega^{0}\right),\left(14, \omega^{1}\right),\left(2, \omega^{2}\right),\left(3, \omega^{3}\right),\left(1, \omega^{4}\right)\right\}$ \\
$\left(0, \omega^{3}\right)$ & $\left\{-,\left(4, \omega^{0}\right),\left(13, \omega^{1}\right),\left(0, \omega^{4}\right),\left(9, \omega^{5}\right)\right\}$ \\
$\left(0, \omega^{4}\right)$ & $\left\{-,\left(8, \omega^{4}\right),\left(15, \omega^{2}\right),\left(8, \omega^{5}\right),\left(11, \omega^{6}\right)\right\}$ \\
$\left(0, \omega^{5}\right)$ & $\left\{-,\left(12, \omega^{5}\right),\left(16, \omega^{3}\right),\left(15, \omega^{7}\right),\left(17, \omega^{6}\right)\right\}$ \\
$\left(0, \omega^{6}\right)$ & $\left\{-,\left(0, \omega^{7}\right),\left(9, \omega^{6}\right),\left(19, \omega^{4}\right),\left(19, \omega^{5}\right)\right\}$ \\
$\left(0, \omega^{7}\right)$ & $\left\{-,\left(2, \omega^{1}\right),\left(10, \omega^{2}\right),\left(12, \omega^{0}\right),\left(17, \omega^{5}\right)\right\}$ \\
\hline \hline$C_{1}:$ & $\left\{\left(15, \omega^{7}\right),\left(2, \omega^{3}\right),\left(14, \omega^{0}\right),\left(5, \omega^{5}\right),\left(11, \omega^{4}\right)\right\}$ \\
$R_{1}:$ & $\left\{\left(11, \omega^{5}\right),\left(5, \omega^{2}\right),\left(5, \omega^{7}\right),\left(13, \omega^{6}\right),\left(7, \omega^{1}\right)\right\}$ \\
\hline
\end{tabular}

Then, for $1 \leqslant i \leqslant 4$, let $S_{i}=S_{1} \cdot\left(1, \omega^{2(i-1)}\right), R_{i}=R_{1} \cdot\left(1, \omega^{2(i-1)}\right), C_{i}=C_{1} \cdot\left(1, \omega^{2(i-1)}\right)$ and $A\left(S_{i}\right)=A\left(S_{1}\right) \cdot\left(1, \omega^{2(i-1)}\right)$. It is an easy matter to verify that $\left(\cup_{i=1}^{4} S_{i}, \cup_{i=1}^{4} R_{i}, \cup_{i=1}^{4} C_{i}\right)$ is the required intransitive starter and $A(S)=\cup_{i=1}^{4} A\left(S_{i}\right)$ is the corresponding adder. Similarly with Lemma 9 , the twenty points from $\mathbb{Z}_{20} \times\{\infty\}$ can be distributed to the five blocks of size four in each $S_{i}$ for $1 \leqslant i \leqslant 4$ in an arbitrary way. So we still use the symbol "-" to denote any point from $\mathbb{Z}_{20} \times\{\infty\}$.

Now we are in a position to complete the existence of NGBTDs with block size five.

Theorem 11. For any positive integer $m$, there exists an $\operatorname{NGBTD}(5, m)$.

Proof : By Theorem 1, we need only to show an $\operatorname{NGBTD}(5, m)$ exists for each $m \in$ $\{15,32,40,45\}$.

An $\operatorname{NGBTD}(5,15)$ is given in Lemma 5. For $m \in\{32,40,45\}$, we apply Theorem 2 with $k=5, w=0$ and other suitable parameters displayed in the following table to obtain the desired $\operatorname{NGBTD}(5, m)$.

\begin{tabular}{ccccc}
\hline$m$ & $g$ & $h$ & $u$ & the source of an FGDRP $\left(k, g^{u}\right)$ \\
\hline 32 & 20 & 4 & 8 & Lemma 9 \\
40 & 20 & 4 & 10 & Lemma 10 \\
45 & 25 & 5 & 9 & Lemma 7 \\
\hline
\end{tabular}

\section{Applications to Constant Composition Codes}

Let $Q=\left\{a_{t}: 0 \leqslant t \leqslant m-1\right\}$ be an arbitrary alphabet set with $m$ elements. A code $C \subseteq Q^{n}$ over $Q$ with size $M$ and minimum distance $d$ is referred to as a constant composition code (CCC), or an $\left(n, M, d,\left[w_{0}, w_{1}, \cdots, w_{m-1}\right]\right)_{m}$-CCC, if each codeword has 
precisely $w_{i}$ occurrences of $a_{i}$ for any $i(0 \leqslant i \leqslant m-1)$. Here the definition implies $n=\sum_{0 \leqslant i \leqslant m-1} w_{i}$.

Since the constant composition $\left[w_{0}, w_{1}, \cdots, w_{m-1}\right]$ is essentially an unordered multiset, we usually write it in an exponential notation: a constant composition $\left[a_{1}^{u_{1}} a_{2}^{u_{2}} \cdots a_{s}^{u_{s}}\right]$ indicates $u_{i}$ occurrences of $a_{i}$ for $1 \leqslant i \leqslant s$ for brevity. We denote the maximum size $M$ of an $\left(n, M, d,\left[w_{0}, w_{1}, \cdots, w_{m-1}\right]\right)_{m^{-}} \mathrm{CCC}$ by $A_{m}\left(n, d,\left[w_{0}, w_{1}, \cdots, w_{m_{1}}\right]\right)$. A CCC with this size is called optimal. The following upper bound was established by Luo et al. [6].

Theorem 12. [6] If $n d-n^{2}+\left(w_{0}^{2}+w_{1}^{2}+\cdots+w_{m-1}^{2}\right)>0$, then

$$
A_{m}\left(n, d,\left[w_{0}, w_{1}, \cdots, w_{m_{1}}\right]\right) \leqslant \frac{n d}{n d-n^{2}+\left(w_{0}^{2}+w_{1}^{2}+\cdots+w_{m-1}^{2}\right)} .
$$

The study of optimal CCCs has attracted extensive attention due to their numerous applications (see, for example, $[3,6]$ and the references therein). Particularly, Ding and Yin [3] presented a combinatorial characterization of constant composition codes and established an equivalent relationship between CCCs and a class of designs called generalized doubly resolvable packings (GDRPs) which are defined below.

Let $X$ be a set of $v$ elements (called points) and $\mathcal{A}$ be a collection of subsets (called blocks) of $X$. Then the pair $(X, \mathcal{A})$ is called a $\lambda$-packing of order $v$, if every pair of distinct points of $X$ occurs in at most $\lambda$ blocks. Furthermore, it is termed a generalized doubly resolvable packing (GDRP), if the blocks of $\mathcal{A}$ can be arranged into an $m \times n$ array $\mathcal{R}$ which satisfies the following properties:

(1) Each cell of $\mathcal{R}$ is either empty or contains one block;

(2) For $0 \leqslant i \leqslant m-1$, the blocks in row $i$ of $\mathcal{R}$ form a $w_{i}$-parallel class, that is, every point occurs in exactly $w_{i}$ blocks;

(3) The blocks in every column of $\mathcal{R}$ form a parallel class, that is, every point occurs in exactly one block.

We denote such a GDRP by a $\operatorname{GDRP}(m \times n, \lambda ; v)$. The multiset $T=$ $\left\{w_{0}, w_{1}, \cdots, w_{m-1}\right\}$ is called the type of the GDRP. For more details, the interested reader may refer to $[3,11,12]$.

Theorem 13. [3, 12] The existence of a $G D R P(m \times n, \lambda ; v)$ of type $\left\{w_{0}, w_{1}, \cdots, w_{m-1}\right\}$ is equivalent to an $\left(n, M, d,\left[w_{0}, w_{1}, \cdots, w_{m-1}\right]\right)_{m}-C C C$, where $M=v$ and $d=n-\lambda$.

Theorem 14. If there exists an $\operatorname{NGBTD}(k, m)$, then there exists an optimal $(k m+1, k m+$ $\left.1, k(m-1)+2,\left[1^{1} k^{m}\right]\right)_{m+1}-C C C$.

Proof : It is readily checked that an $\operatorname{NGBTD}(k, m)$ is a $\operatorname{GDRP}(m \times(k m+1), k-1 ; k m+$ $1)$ of type $\{1, k, \cdots, k\}$. By Theorem 13 , we have an $\left(n, M, d,\left[w_{0}, w_{1}, \cdots, w_{m-1}\right]\right)_{m}$ CCC with $n=M=k m+1, d=k(m-1)+2, w_{0}=1, w_{1}=w_{2}=\cdots=w_{m-1}=k$. In addition, 
by Theorem 12, we have

$$
\begin{aligned}
& A_{m}\left(k m+1, k(m-1)+2,\left[1^{1} k^{m}\right]\right) \\
& \leqslant \frac{(k m+1)(k(m-1)+2)}{(k m+1)(k(m-1)+2)-(k m+1)^{2}+\left(1^{2}+k^{2}+\cdots+k^{2}\right)} \\
& =\frac{(k m+1)(k(m-1)+2)}{(k m+1)(1-k)+\left(1+m k^{2}\right)} \\
& =k m+1
\end{aligned}
$$

Hence the obtained CCC is optimal. Then the proof is complete.

Theorem 15. Let $m, k$ be integers satisfying $m \geqslant 1,2 \leqslant k \leqslant 5$ and $(k, m) \notin(3,3)$. Then there exists an optimal $\left(k m+1, k m+1, k(m-1)+2,\left[1^{1} k^{m}\right]\right)_{m+1}-C C C$.

Proof : By Theorem 1, 4 and 11, there exists an $\operatorname{NGBTD}(k, m)$ for any integers $m$ and $k$ where $m \geqslant 1,2 \leqslant k \leqslant 5$ and $(k, m) \notin(3,3)$. Then the conclusion follows from Theorem 14.

\section{Acknowledgement}

The authors thank the referees for their valuable comments and suggestions.

This work is supported by NSFC under Grants No. 10801064, 11001109, and 10926103, and by the Program for Innovative Research Team of Jiangnan University.

\section{References}

[1] C. J. Colbourn and J. H. Dinitz. The CRC Handbook of Combinatorial Designs. CRC Press, Boca Raton, FL, 2007.

[2] C. J. Colbourn, E. R. Lamken, A. C. H. Ling and W. H. Mills. The existence of Kirkman squares-doubly resolvable $(v, 3,1)$-BIBDs. Des. Codes Cryptogr. 26:169196, 2002.

[3] C. Ding and J. Yin. Combinatorial constructions of optimal constant composition codes. IEEE Trans. Inf. Theory, 51:3671-3674, 2005.

[4] E. R. Lamken. On near generalized balanced tournament designs, Discrete Math., 97:279-294, 1991.

[5] E. R. Lamken and S. A. Vanstone. Orthogonal resolutions in odd balanced tournament designs. Graphs Combin., 4:241-255, 1988.

[6] Y. Luo, F. W. Fu, A. J. Han Vinck and W. Chen. On constant composition codes over $Z_{q}$. IEEE Trans. Inf. Theory, 49:3010-3016, 2003.

[7] A. Rosa and S. A. Vanstone. Starter-adder techniques for Kirkman squares and Kirkman cubes of small sides. Ars Combinatoria, 14:199-212, 1982.

[8] X. Shan. Near generalized balanced tournament designs with block sizes 4 and 5. Sci. China Ser. A, 50:1382-1388, 2007. 
[9] J. Yan. Generalized doubly resolvable packings and the corresponding codes. Ph.D thesis, Suzhou, Suzhou University, 2007.

[10] J. Yan and C. Wang. The existence of FGDRP $\left(3, g^{u}\right)^{\prime}$ 's. Electron. J. Combin., 16(1):\#R34, 2009.

[11] J. Yan and J. Yin. Constructions of optimal $\operatorname{GDRP}(n, \lambda ; v)$ of type $\lambda^{1} \mu^{m-1}$. Discrete Appl. Math., 156:2666-2678, 2008.

[12] J. Yan and J. Yin. A class of optimal constant composition codes from GDRPs. Des. Codes Cryptogr., 50:61-76, 2009.

[13] J. Yin, J. Yan and C. Wang. Generalized balanced tournament designs and related codes. Des. Codes Cryptogr., 46:211-230, 2008. 